\title{
The effect of L-theanine supplementation on the immune system of athletes exposed to strenuous physical exercise
}

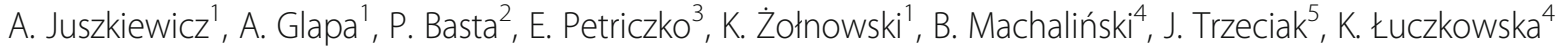 \\ and A. Skarpańska-Stejnborn ${ }^{1 *}$ (D)
}

\begin{abstract}
Background: The aim of this study was to analyze the response of selected components of the immune system in rowers to maximal physical exercise, and to verify if this response could be modulated by supplementation with L-theanine.

Method: The double-blind study included 20 members of the Polish Rowing Team. The subjects were randomly assigned to the supplemented group $(n=10)$, receiving $150 \mathrm{mg}$ of L-theanine extract for 6 weeks, or to the placebo group $(n=10)$. The participants performed a 2000-m test on a rowing ergometer at the beginning (1st examination) and at the end of the supplementation period (2nd examination). Blood samples were obtained from the antecubital vein before each exercise test, 1 min after completing the test, and after a 24-h recovery. Subpopulations of T regulatory lymphocytes (Tregs) (CD4+/CD25+/CD127-), cytotoxic lymphocytes (CTLs) (CD8+/TCRaß+), natural killer (NK)

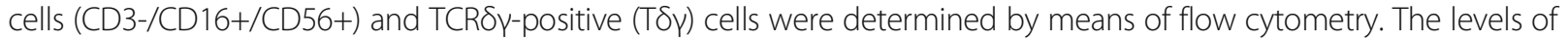
interleukin 2 (IL-2), interleukin 4 (IL-4), interleukin 10 (IL-10), interferon gamma (INF- $\gamma$ ) and total antioxidant capacity (TAC) were determined with commercially available diagnostic kits.

Results: Supplementation with L-theanine contributed to a significant post-exercise decrease in IL-10 concentration, which was reflected by higher values of IL-2 to IL-10 and IFN- $\gamma$ to IL-10 ratios. Moreover, a significant post-recovery decrease in CTL count, Treg to NK and Treg to CTL ratios was observed in the supplemented group.

Conclusion: Despite the decrease in the number of some cytotoxic cells (CTLS) and an increase in the proportion of Tregs to CTLs, supplementation with LTE seems to exert a beneficial effect on a disrupted Th1/Th2 balance in elite athletes, as shown by the decrease in IL-10 concentration.
\end{abstract}

Keywords: L-theanine, Inflammation, Flow cytometry, Cytokine, Strenuous exercise, Rowers

\section{Introduction}

Elite athletes, exposed to extremely large training loads, may develop the so-called overtraining syndrome (OTS) which may inter alia contribute to a decrease in their performance [1, 2]. One consequence of OTS is greater susceptibility to upper respiratory tract infections (URTI), which suggests that overtraining is associated with immune impairment [3]. Recent evidence showed

\footnotetext{
* Correspondence: ankass@poczta.onet.pl

${ }^{1}$ Department of Morphological and Health Sciences, Faculty of Physical Culture in Gorzów Wielkopolski, 13 Estkowskiego Str, 66-400 Gorzów Wielkopolski, Poland

Full list of author information is available at the end of the article
}

that the immune impairment in OTS may be caused by a disruption of Th1/Th2 balance. Human T-helper (Th) lymphocytes are represented by two distinct functional subsets: Th1 cells, causing activation of cell-mediated immunity associated with the elimination of intracellular pathogens and anticancer protection, and Th2 cells, stimulating humoral immunity associated with antibody synthesis. Previous studies demonstrated that strenuous exercise may contribute to cellular immunosuppression and resultant shift in Th1/Th2 balance toward Th2, which results in greater susceptibility to infection, inflammation and overtraining [4-6]. This justifies the inclusion of supplements with established immunomodulatory effects in

(c) The Author(s). 2019 Open Access This article is distributed under the terms of the Creative Commons Attribution 4.0 International License (http://creativecommons.org/licenses/by/4.0/), which permits unrestricted use, distribution, and 
athletes' diet, as an element of a mild and safe intervention aimed at restoration of immune balance.

L-theanine (LTE) is a main amino acid present in tea leaves. LTE is metabolized in kidneys to glutamic acid and ethylamine [7]. The latter is an alkylamine, and aside from the tea leaves, can also be found (albeit at a lower amount) in other products, e.g. mushrooms, apples, red wine, to mention a few [8]. Previous studies showed that LTE may modulate immunity, regulating secretion of Th1 and Th2 cytokines. Animals supplemented with LTE presented with higher serum concentrations of interleukin-2 (IL-2) and interferon gamma (IFN- $\gamma$ ) [9]. Another study showed that administration of LTE conditioned $\mathrm{T} \gamma \delta$ lymphocytes for stronger response to the stimulus triggering cytotoxic reaction. Drinking black tea contributed to a significant increase in the secretion of IFN- $\gamma$ by $\mathrm{T} \gamma \delta$ lymphocytes from healthy volunteers, after in vitro stimulation with bacterial antigens [8]. In turn, addition of ethylamine to the culture of peripheral blood mononuclear cells obtained from healthy volunteers resulted a 15 -fold increase in $\mathrm{T} \gamma \delta$ count [10]. At low, micromolar, concentrations alkylamines maintain $\mathrm{T} \gamma \delta$ readiness for a rapid response to invasion of pathogenic or opportunistic bacteria; the number of $\mathrm{T} \gamma \delta$ cells and the synthesis of antibacterial cytokines (among them IFN- $\gamma$ ) were shown to increase substantially following exposure to bacterial antigens. However, without antigenic stimulation, markedly higher, millimolar, concentrations of alkylamines are required to induce the proliferation of $\mathrm{T} \gamma \delta$ cells [8].

Murakami et al. [11] analyzed the effects of supplementation with LTE and cystine on some immune functions in athletes after a long run. While a decrease in lymphocyte count was documented after the run in non-supplemented controls, no similar effect was observed in the supplemented group. The same study showed a statistically significant increase in the activity of natural killer (NK) cells; although this effect was observed in both groups, it was markedly more evident in the athletes supplemented with LTE and cystine. According to Kawada et al. [12], the post-exercise activation of NK cells in well-trained men supplemented with LTE and cystine was significantly stronger than in non-supplemented controls. In both studies mentioned above, LTE was administered together with cystine; this regimen was chosen based on the results of a previous animal study in which mice received cystine and/or LTE. The study showed that stimulation with theanine and cystine contributed to a substantial increase in the synthesis of IgG following antigenic stimulation. Moreover, animals supplemented with LTE and cystine presented with significantly higher values of IL-10 to IFN- $\gamma$ ratio. In contrast, the values of this parameter in mice that received LTE alone were lower than at the baseline, which probably resulted from a significant decrease in IL-10 level, observed already within $24 \mathrm{~h}$ of the supplementation [13].

As shown above, previous studies demonstrated that both cystine and intense physical exercise may promote a shift in Th1/Th2 balance toward Th2. Thus, we hypothesized that supplementation with LTE may promote Th1 response and attenuate Th2 response in elite athletes. One method for functional assessment of the Th1/Th2 balance is determination of Th1 (e.g. INF- $\gamma$, IL-2) and Th2 cytokine (e.g. IL-4, IL-10) levels. However, to accurately estimate the status of Th1/Th2 balance, the levels of cytokines produced by Th1 and Th2 lymphocytes should be interpreted together, since an increase in Th1 cytokine concentration may be frequently counterbalanced by an increase/decrease in Th2 cytokine level, and vice versa $[14,15]$. Therefore, the analysis of a shift in Th1/Th2 balance triggered by strenuous physical exercise (an extreme stressor) and supplementation (LTE) based on the values of INF- $\gamma$ to IL-4, INF- $\gamma$ to IL-10, IL-2 to IL-4 and IL-2 to IL-10 ratios would be more accurate than merely the analysis of changes in concentrations of individual cytokines. Another parameter to be considered during the evaluation of immune balance after strenuous physical exercise is the number of cytotoxic cells (e.g. NK, CLT, T $\gamma \delta$ ) and T-regulatory lymphocytes (Tregs).

Since other authors demonstrated that various lymphocyte fractions can interact with one another, either directly or via synthesized cytokines, we searched for sufficiently accurate markers of those relationships. Based on the observations published by other researchers [16-19] who demonstrated that Treg to effector T-cell ratios (Treg to T $\gamma \delta$, Treg to NK, Treg to CTL, Treg to NK + TCR + CLT) are more accurate measures of the suppressive effects exerted by the regulatory lymphocytes than a simple cytometry, we also considered those indices in our study. To the best of our knowledge, this study was the first one to use such comprehensive approach to the analysis of exercise-induced changes in Th1/Th2 balance in elite athletes.

\section{Materials and methods Study population}

The study included 20 men, all members of the Polish Rowing Team (16 heavy-weight and 4 light-weight rowers). Basic characteristics and sport classes of the athletes are presented in Table 1. The study was conducted between March and May, during a 6-week training camp scheduled between the preparatory and competitive phase of the yearly training cycle. The characteristics of the training profile, such as its intensity, volume (in minutes) and type (specific, i.e. rowing: endurance, technical, speed, etc., and nonspecific: jogging, 
Table 1 Basic characteristics of the study groups

\begin{tabular}{lll}
\hline Parameters & $\begin{array}{l}\text { Supplemented group } \\
(n=10)\end{array}$ & $\begin{array}{l}\text { Placebo group } \\
(n=10)\end{array}$ \\
\hline Age (years) & $21.0 \pm 0.91$ & $20.5 \pm 1.08$ \\
Body weight $(\mathrm{kg})$ & $88.9 \pm 7.02$ & $85.7 \pm 10.40$ \\
Body height $(\mathrm{cm})$ & $191.2 \pm 3.03$ & $187.9 \pm 5.62$ \\
Duration of training (years) & $7.5 \pm 1.5$ & $6.3 \pm 1.5$ \\
\hline
\end{tabular}

Values represent means \pm standard deviations. No statistically significant differences were found for all intergroup comparisons $(P>0.05)$

strength) were recorded on a daily basis. The intensity of the training was classified based on the lactate acid (LA) threshold $(4 \mathrm{mmol} / \mathrm{L})$, as extensive (below the LA threshold), highly intensive (above the LA threshold), and extremely intensive (control tests) (Table 2).

\section{Food intake}

Throughout the study period, the athletes were accommodated at an Olympic Training Center, whereby they had all their meals. Their regular menu consisted of a mixed diet, providing the recommended dietary allowance (RDA) of carbohydrates, proteins, fats and micronutrients (vitamins and minerals), in line with the Polish Nutrition Society guidelines [20]. Daily intakes of food, calories, fruits and vegetables were the same throughout the study period. None of the athletes drank more than two cups of tea per day. Average
L-theanine content per single cup of tea approximates 7.9-24.2 mg [21].

The study subjects declared that they had ceased all drugs, medications and dietary supplements at least 2 weeks before the experiment, and did not use them throughout the entire study period.

\section{Experimental procedure}

Athletes who were randomized to the supplemented group $(n=10)$ received gelatin capsules with $150 \mathrm{mg}$ L-theanine extract, manufactured by Nanga (Złotów, Poland). The subjects were asked to take two capsules per day for 6 weeks. The dose was selected based on published data $[22,23]$. Athletes randomized to the placebo group $(n=10)$ received visually identical capsules with cornstarch (150 mg per capsule).

\section{Training program}

Training volumes (expressed in minutes per day) during a week preceding the 1st and the 2nd examination are shown in Table 2, separately for extensive rowing, intensive rowing, kilometers and extensive non-specific training. During the load training phase (before the 1st examination), the training volume amounted to 970 min per week, including approximately $42.9 \%$ of extensive rowing, $10.2 \%$ of non-specific training (e.g. power training) and $20.1 \%$ of intensive rowing. Total training volume before the 2 nd examination was $960 \mathrm{~min} \cdot$ per week and included approximately

Table 2 Training schedule for the week preceding blood sampling during the 1st and the 2nd examination

Table 2 Training schedule for the week the 1st examination
Days beceding blood sampling during the 1st and the 2 na

Total training time, min/day
Time rowed, min/day
Distance rowed, km/day
Training for force development, min/day
Extensive endurance rowing training time, min/day
High intensity endurance rowing training time, min/day
Unspecific training (running, etc.), min/day

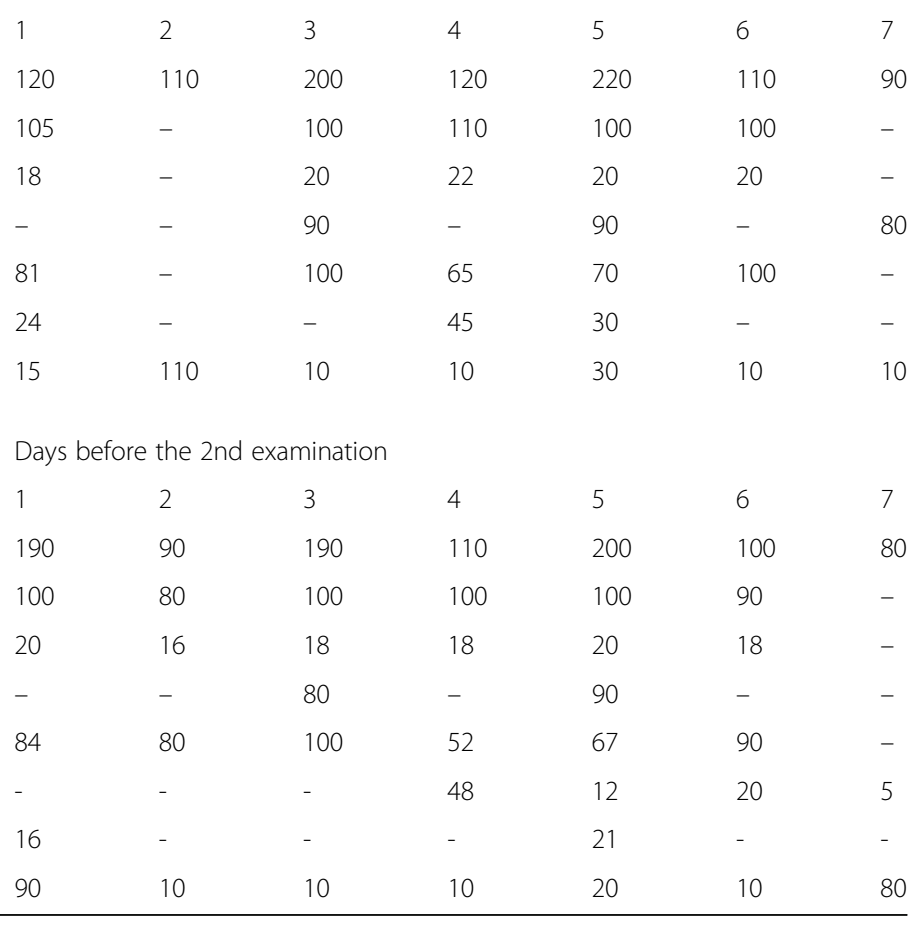

Total training time, min/day

Time rowed, min/day

Distance rowed, $\mathrm{km} /$ day

Training for force development, min/day

Extensive endurance rowing training time, min/day

High intensity endurance rowing training time, min/day

Very high intensity endurance rowing training time, min/day

Unspecific training (running, etc.), min/day
Days before the 2 nd examination

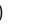

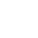
80

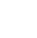

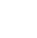


$48.2 \%$ of extensive rowing, $7.3 \%$ of intensive rowing (with $3.9 \%$ of maximum-intensity control tests).

\section{Rowing performance test}

The athletes performed a controlled 2000-m time trial on the first day (before the supplementation) and at the end of the training camp (after the supplementation). Each subject had to cover the 2000-m distance on a rowing ergometer (Concept II, USA) in the shortest time possible. Because the results of both tests were taken into consideration during selection to the championship team, the athletes were motivated well to perform both tests at a maximal effort. Before each test, the subjects performed a 5-min individual warm-up on the ergometer.

\section{Sample treatment}

Blood samples from the antecubital vein were collected to tubes with dipotassium ethylene diamine tetra-acetic acid $\left(\mathrm{K}_{2}\right.$ EDTA $)$ as an anticoagulant. Blood was collected before each 2000-m test (after 7- to 8-h overnight fast), 1 min after completing the test, and after a 24-h recovery. The list of analyzed parameters included white blood cell (WBC) counts and percentages of lymphocytes and granulocytes, all determined with MYTHIC 18 Hematology Analyzer (Orphee Medical, Geneva, Switzerland). The samples collected to tubes with the anticoagulant were centrifuged for $10 \mathrm{~min}$ at $2200 \mathrm{rpm}$. After removing the plasma and adding 1x Lysing Buffer (BD Biosciences), the samples were incubated in darkness for $15 \mathrm{~min}$. Then, PBS buffer was added, and the cells were washed twice to remove all erythrocytes. Blood samples collected to the tubes without additives were centrifuged for $10 \mathrm{~min}$ at $3500 \mathrm{rpm}$, and the sera were stored at $-80^{\circ} \mathrm{C}$ until the analysis. Moreover, capillary blood samples from the earlobe were collected before and after each exercise test, to assess LA level.

\section{Measurements}

Cytometric analysis of lymphocyte subpopulations: Tregs (CD4+/CD25+/CD127-), CTLs (CD8+/TCR $\alpha \beta+)$, NK cells (CD3-/CD16+/CD56+) and T $\gamma \gamma$ cells was conducted after their labeling with fluorochrome-conjugated antibodies from BD Biosciences. The cells obtained after hypotonic lysis of peripheral blood were incubated in darkness at room temperature for $20 \mathrm{~min}$ with the respective antibody at a concentration specified by the manufacturer, to identify each lymphocyte subpopulation (Table 3 ). Then, the cells were washed twice with PBS buffer and left in darkness in $3.7 \%$ formaldehyde solution for $10 \mathrm{~min}$. Subsequently, the cells were again washed with PBS buffer, and $100 \mu \mathrm{l}$ of DAPI solution $(1 \mathrm{mg} / \mathrm{ml}$, Thermo Fisher Scientific) were added to stain cell nuclei. Then, the cells were incubated in darkness at room temperature for $5 \mathrm{~min}$, washed twice, and suspended in $250 \mu \mathrm{L}$ of PBS
Table 3 Antibodies used for identification of lymphocyte subpopulations

\begin{tabular}{ll}
\hline Lymphocyte subpopulation & Antibody \\
\hline Regulatory T lymphocytes & FITC Mouse Anti-Human CD4 \\
& PE Mouse Anti-Human CD25 \\
& Alexa Fluor 647 Mouse anti-Human CD127 \\
Cytotoxic lymphocytes & APC Mouse Anti-Human CD8 \\
& FITC Mouse Anti-Human TCR a \\
NK cells & FITC Mouse Anti-Human CD3 \\
& PE Mouse Anti-Human CD16 \\
& APC Mouse Anti-Human CD56 \\
TCR $/ Y$ lymphocytes & FITC Mouse Anti-Human TCRס/Y \\
\hline
\end{tabular}

buffer. After labeling, the cells were analyzed with LSRII flow cytometer (BD Biosciences) coupled with BD FACSDiva software.

Serum concentrations of interleukins: IL-2, IL-4 and IL-10 were measured using a commercially available enzyme immunoassays (ELISA; Abcam, Cambridge, UK) with detection ranges of $1.87-60 \mathrm{pg} / \mathrm{ml}, 0.31-10 \mathrm{pg} / \mathrm{ml}$ and $1.56-50 \mathrm{pg} / \mathrm{ml}$, respectively. Serum levels of interferon gamma (IFN- $\gamma$ ) were quantified using a commercially available enzyme immunoassay (ELISA; Quantikine HS, R\&D Systems, Minneapolis, USA) with a detection range of $15.6-1000 \mathrm{pg} / \mathrm{ml}$.

Total antioxidant capacity (TAC), considered a marker of plasma antioxidant capacity, was assessed with a commercially available kit (LDN Labor Diagnostika Nordhorn, Germany) with an assay range of $0.375-3 \mathrm{mmol} / \mathrm{l}$. Concentration of LA in capillary blood was determined immediately after sampling, using a commercially available kit (Dr Lange, Germany); LA concentrations were expressed in $\mathrm{mmol} / \mathrm{l}$. The coefficients of variation for all assays were $<11 \%$.

\section{Statistical analysis}

Statistical analyses were conducted with STATISTICA v. 13.1 software package (StatSoft, Cracow, Poland). All parameters were compared using 2 (supplementation: supplemented vs. placebo) $\times 3$ (exercise: pre-exercise vs. post-exercise vs. post-recovery) repeated measures analysis of variance (ANOVA). Normal distribution of the study variables was verified with Shapiro-Wilk test. Whenever the result of ANOVA was statistically significant, Fisher's post-hoc test was conducted to identify the source of significant differences. Anthropometric characteristics of the study groups were compared with unpaired Student's $t$-test. Except the rowing time, the results of the 2000- $\mathrm{m}$ tests performed before and after the supplementation were compared with paired Student's $t$-test, and intergroup comparisons were conducted with unpaired Student's $t$-test. The results of the 2000-m simulated rowing test were subjected to one-way ANOVA. Statistical characteristics of the study variables are presented as 
means \pm standard deviations (SD), and the threshold of statistical significance for all tests was set at $p<0.05$.

\section{Results}

Athletes from the supplemented group and the placebo group did not differ significantly in terms of their mean age, body height, body weight and years of training (Table 1).

No significant intragroup differences were found in mean power output and total run time during the 2000- $\mathrm{m}$ test performed at the beginning and at the end of the training camp (1st and 2nd examination, respectively). Furthermore, no significant intragroup differences in the pre- and post-supplementation blood LA levels were documented (Table 4).

During the 1st examination, a significant post-exercise increase in IL-2 level was observed in both study groups (Fig. 1a). However, no statistically significant exercise-induced changes in the concentration of this cytokine were documented during the 2nd examination. Furthermore, this parameter turned out to be independent of L-theanine supplementation (ANOVA, main effect, $p=0.981$ ).

Changes in IL-10 levels in the study athletes are presented in Fig. 1b. During the 1st examination, the post-recovery levels of IL-10 in both study groups were significantly lower than at the baseline and immediately after the exercise test. Although supplementation with L-theanine did not exert a significant effect on IL-10 level (main effect, $p=0.943$ ), during the 2nd examination, the post-recovery concentration of this cytokine in the supplemented group was significantly lower than at the baseline and immediately after the exercise.

The levels of IL-4 at various study timepoints are presented in Fig. 1c. This parameter turned out to be modulated by neither physical exercise (main effect, $p=0.662$ ) nor supplementation (main effect, $p=0.508$ ).

No statistically significant changes in IFN- $\gamma$ levels were observed during the 1st examination (Fig. 1d). In contrast, a post-exercise increase in this parameter, which also persisted after a 24-h recovery, was demonstrated in both groups during the 2nd examination. Statistical analysis showed that supplementation with L-theanine did not exert a significant effect on IFN- $\gamma$ levels (main effect, $p=0.172$ ).

The values of proinflammatory to anti-inflammatory cytokine ratios are presented in Fig. 2. Supplementation with L-theanine did not influence significantly any of the analyzed indices of Th1/Th2 balance. However, ANOVA demonstrated that physical exercise exerted significant effects on the values of all ratios (main effect $p<0.001$ for all analyzed parameters).

During the 1st examination, physical exercise contributed to a significant increase in the values of IL-2 to IL-4 and IL-2 to IL-10 ratios in both groups, and this effect also persisted after a 24-h recovery (Fig. 2a and b). During the 2nd examination, the post-recovery values of IL-2 to IL-4 ratio in the supplemented group, but not in the placebo group, were significantly higher than at the baseline.

Statistically significant changes in IFN- $\gamma$ to IL-4 ratio were demonstrated solely during the 1st examination when the post-recovery values of this parameter in both study groups were significantly higher than at the baseline and immediately after the exercise (Fig. 2c). In turn, statistically significant changes in IFN- $\gamma$ to IL-10 ratio were observed solely during the 2nd examination, when athletes from both groups showed a significant post-exercise increase in this parameter. After a 24-h recovery, the values of IFN- $\gamma$ to IL-10 ratio in the supplemented group, but not in the placebo group, were still significantly higher than at the baseline (Fig. 2d).

Treg counts determined during the 1st and the 2nd examination are shown in Fig. 3a. ANOVA revealed a significant main effect of exercise on Treg count $(p<0.001)$. During the 2nd examination, the post-recovery Treg counts in athletes from both groups were significantly higher than at the baseline $(p<0.05)$. However, ANOVA demonstrated that supplementation with L-theanine did not exert a statistically significant effect on Treg count (main effect, $p=0.149$ ).

Table 4 Changes in $2000 \mathrm{~m}$ rowing ergometer performance before and after supplementation with LTE

\begin{tabular}{|c|c|c|c|c|}
\hline \multirow[t]{2}{*}{ Parameters } & \multicolumn{2}{|c|}{$\begin{array}{l}\text { Supplemented group } \\
(n=10)\end{array}$} & \multicolumn{2}{|l|}{$\begin{array}{l}\text { Placebo group } \\
(n=10)\end{array}$} \\
\hline & Before & After & Before & After \\
\hline Power (Watt) & $436 \pm 28.5$ & $445 \pm 17.52$ & $401 \pm 31.48$ & $411 \pm 36.69$ \\
\hline$\left(\mathrm{W} \times \mathrm{kg}^{-1}\right)$ & $5.01 \pm 0.37$ & $5.02 \pm 0.28$ & $4.81 \pm 0.44$ & $4.93 \pm 0.22$ \\
\hline $\mathrm{LA}_{\min }\left(\mathrm{mmol} \times \mathrm{L}^{-1}\right)^{\mathrm{a}}$ & $1.6 \pm 0.37$ & $1.5 \pm 0.81$ & $1.8 \pm 0.73$ & $1.6 \pm 0.25$ \\
\hline$L A_{\max }\left(m m o l \times L^{-1}\right)^{a}$ & $15.1 \pm 2.31$ & $11.6 \pm 2.42$ & $14.1 \pm 3.18$ & $12.3 \pm 4.13$ \\
\hline Time (s) & $371.3 \pm 8.47$ & $368.7 \pm 5.22$ & $382.1 \pm 12.98$ & $379.2 \pm 11.78$ \\
\hline
\end{tabular}

Values represent means \pm standard deviations. ${ }^{a} \mathrm{LA}$, lactate. No statistically significant differences were found between the pre- and post-supplementation results $(P<0.05)$ 


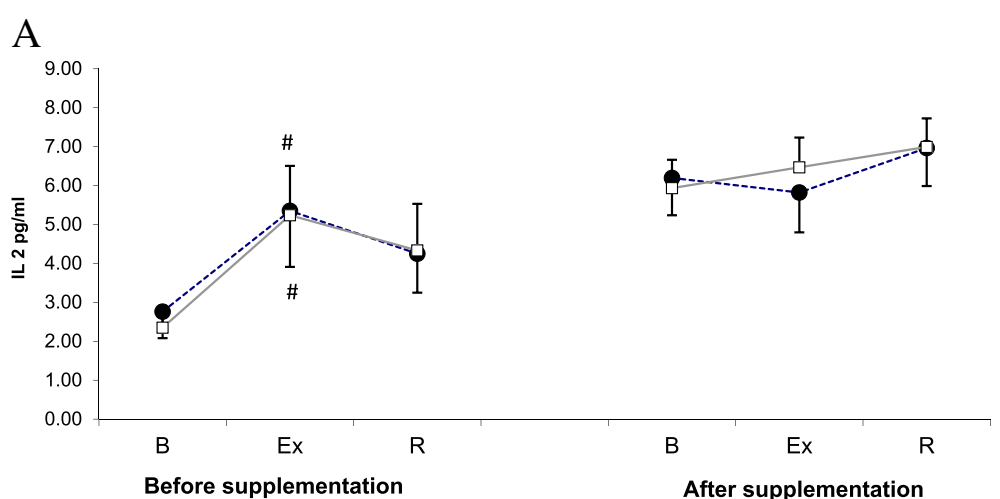

B

Before supplementation

After supplementation

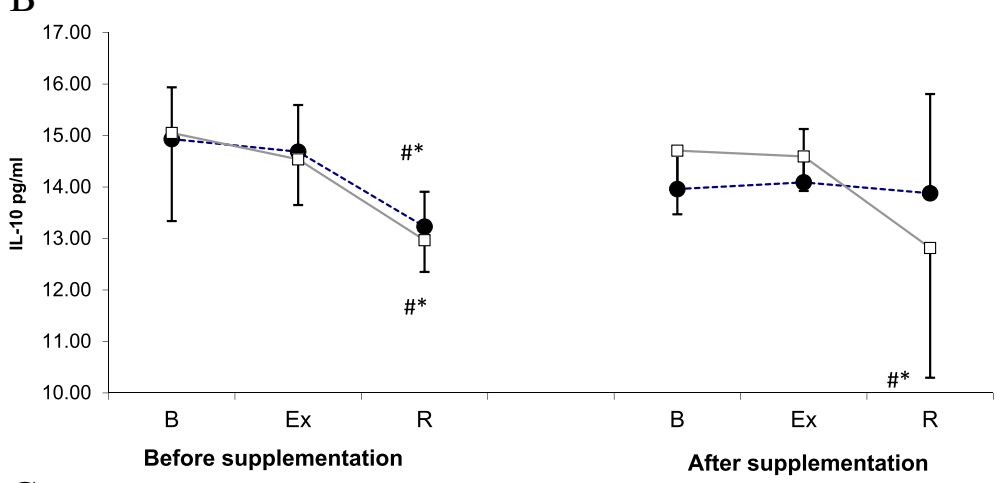

C

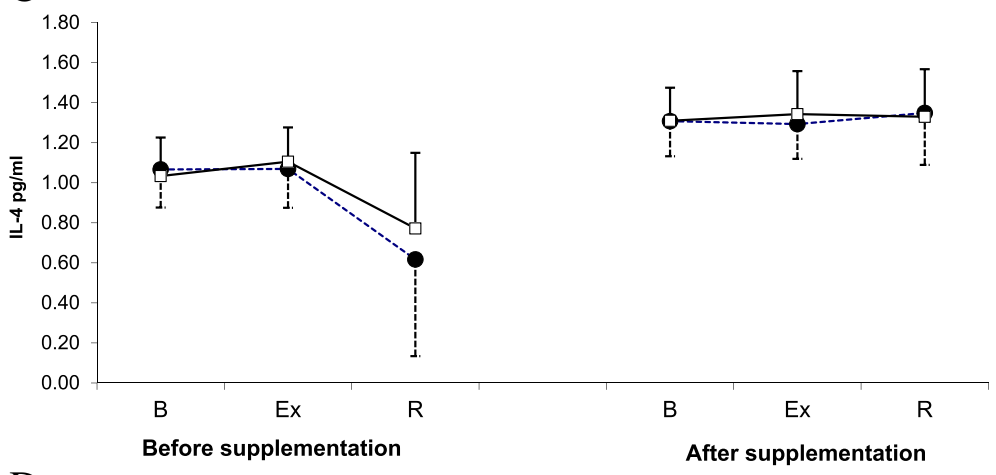

$\mathrm{D}$

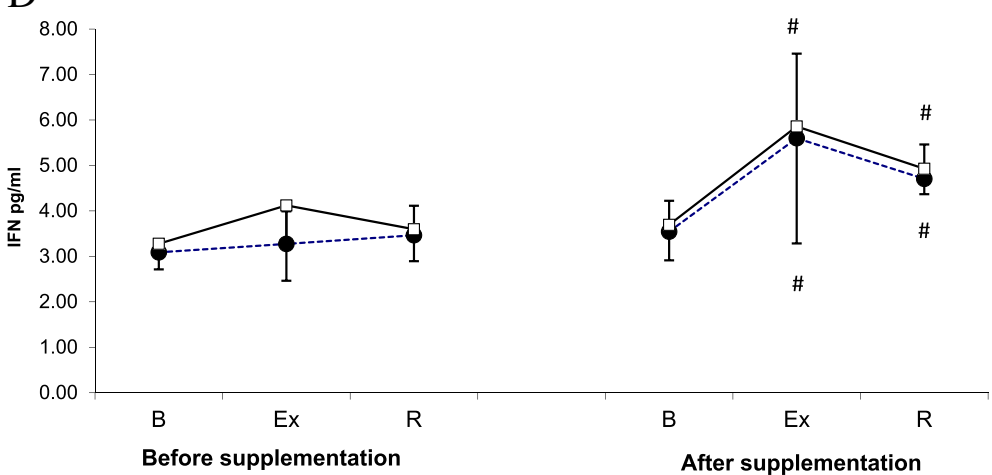

Fig. 1 Changes in IL-2 (a), IL-10 (b), IL-4 (c), and IFN-gamma (d) levels during exercise tests performed prior to and after supplementation with LTE (mean \pm SD). Note. IL = interleukins; INF $\gamma=$ interferon gamma; $\square-$ SUPPL = supplemented group; $\bullet-P L A=$ placebo group; $B=$ baseline; Ex = post-exercise; $R$ = after a 1-day recovery; \# - significantly different compared to the baseline level; * - significantly different compared to the post-exercise level 


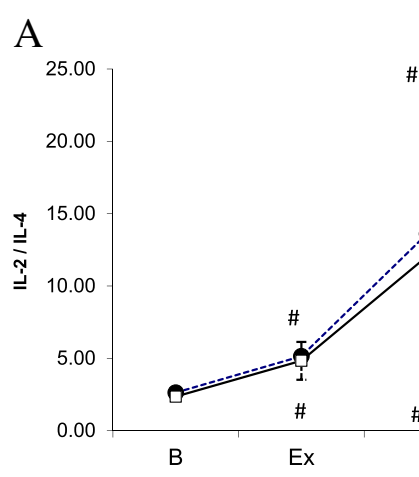

B

Before supplementation

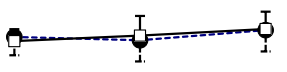

$\mathrm{R}$

Ex $\mathrm{R}$

After supplementation

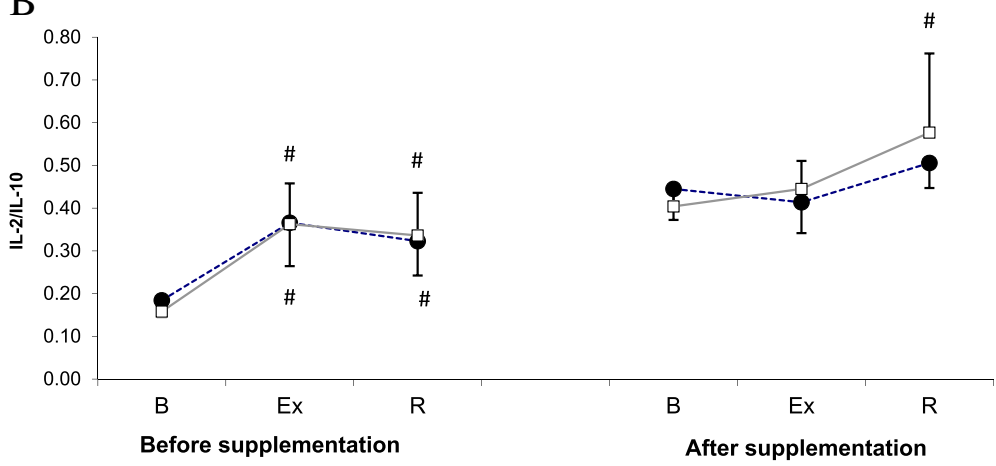

$\mathrm{C}$

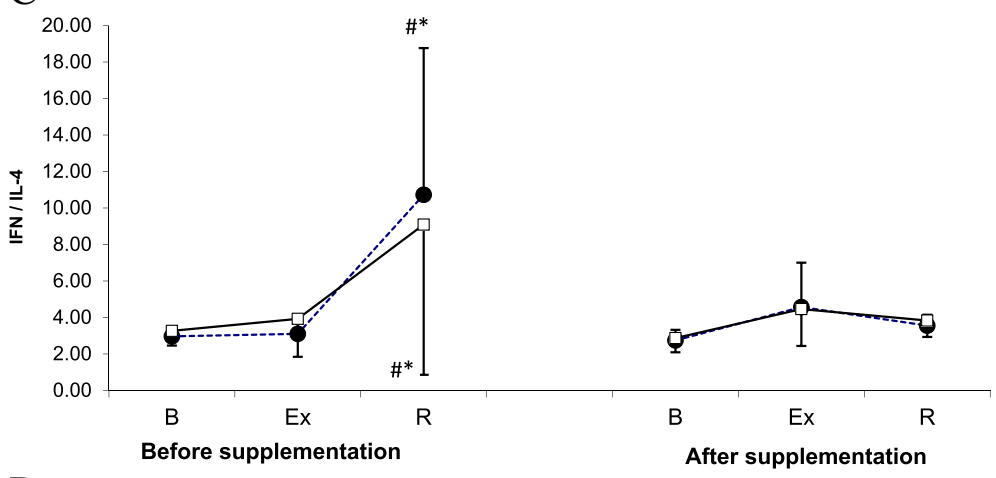

$\mathrm{D}$

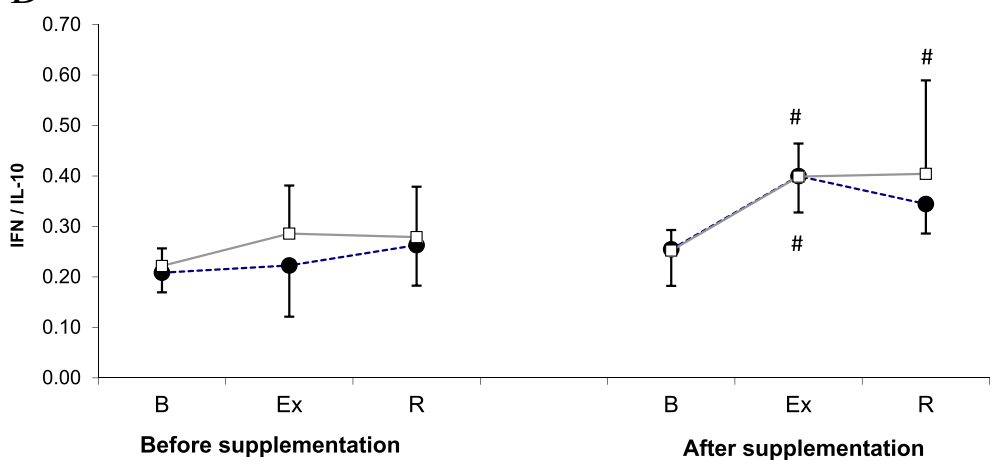

Fig. $2 \mathrm{IL}-2 / \mathrm{LL}-4(\mathbf{a}), \mathrm{IL}-2 / \mathrm{LL}-10(\mathbf{b}), \mathrm{IFN}-\gamma / \mathrm{LL}-4$ (c) and IFN- $\gamma / \mathrm{LL}-10$ (d) ratios during exercise tests performed before and after supplementation with LTE (mean \pm SD). Note. IL = interleukins; INF $\gamma=$ interferon gamma; $\square-$ SUPPL = supplemented group; $\bullet-P L A=$ placebo group; B = baseline; Ex = post-exercise; $R$ = after a 1-day recovery; \# - significantly different compared to the baseline level; ${ }^{*}$ - significantly different compared to the post-exercise level 


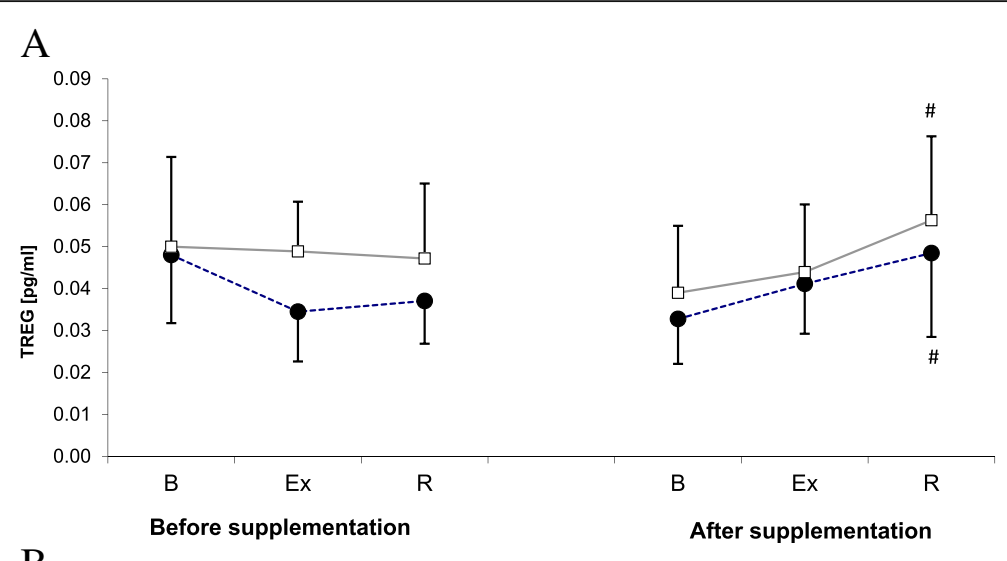

B

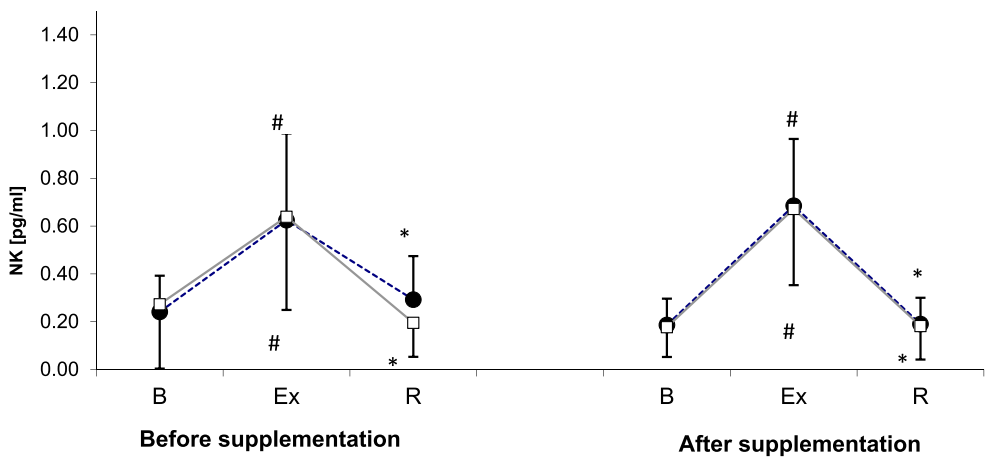

$\mathrm{C}$

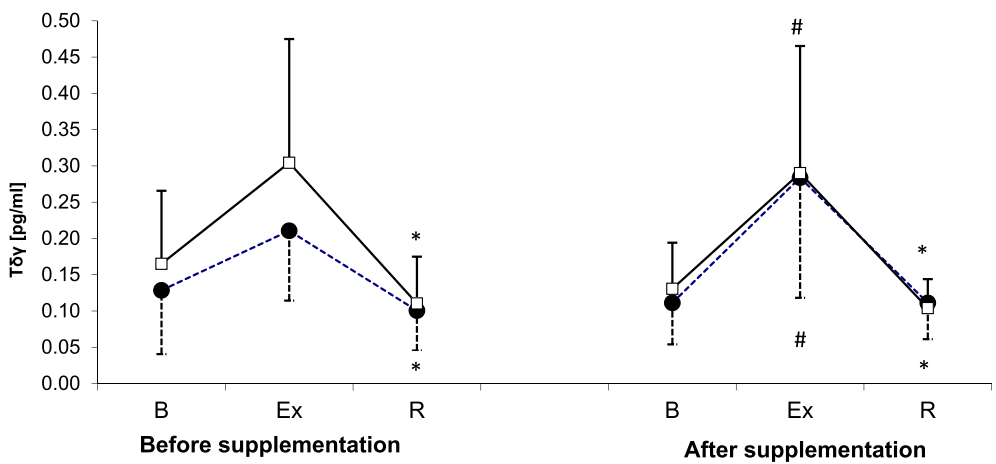

$\mathrm{D}$

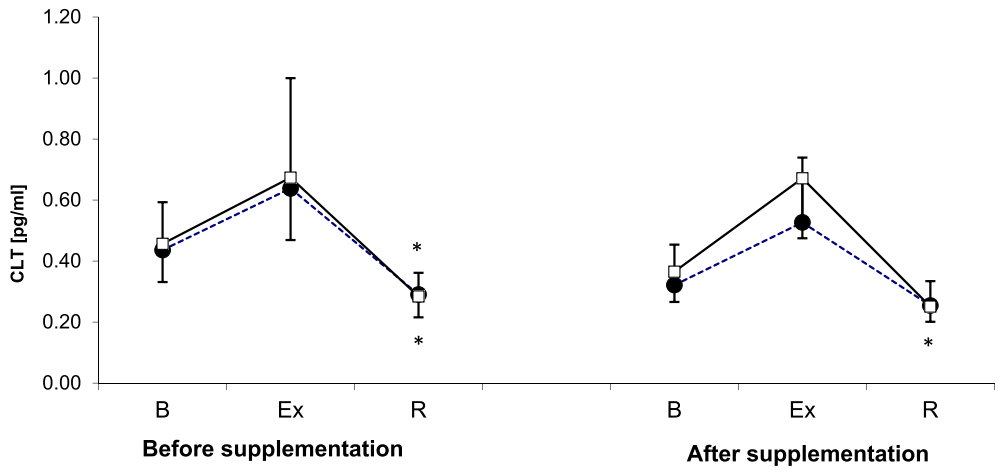

Fig. 3 Changes in Treg (a), NK cell (b), T $\delta \gamma$ cell (c) and CTL (d) counts during exercise tests performed prior to and after supplementation with LTE (mean $\pm S D)$. Note. Tregs = regulatory $T$ cells; NK = natural killer cells; $T \delta \gamma=$ gamma delta $T$ cells; $C L T=$ cytotoxic $T$ lymphocytes; $\square-$ SUPPL = supplemented group; $\bullet-$ PLA = placebo group; B = baseline; EX = post-exercise; $\mathrm{R}=$ after a 1-day recovery; \# - significantly different compared to the baseline level; * - significantly different compared to the post-exercise level 
NK cell counts determined before and after supplementation with L-theanine are shown in Fig. 3b. ANOVA demonstrated a significant main effect of exercise on NK cell count $(p<0.001)$. During both the 1st and the 2nd examination, a significant post-exercise increase in this parameter was observed in both supplemented group and placebo group, followed by a post-recovery normalization at the baseline level.

$\mathrm{T} \delta \gamma$ cell counts in the study subjects are presented in Fig. 3c. Likewise for other lymphocyte subpopulations, ANOVA demonstrated a significant main effect of exercise on T $\delta \gamma$ cell count $(p<0.001)$. During the 1st examination, the post-recovery $\mathrm{T} \delta \gamma$ cell counts in both groups were significantly lower than immediately after the exercise. During the 2nd examination, physical exercise contributed to a significant increase in $\mathrm{T} \delta \gamma$ cell counts in both groups, with subsequent return of this parameter to its baseline levels following a 24-h recovery. The main effect of the supplementation on $T \delta \gamma$ cell count did not turn out to be statistically significant $(p=0.156)$.

ANOVA demonstrated that exercise exerted a significant main effect on CTL count $(p<0.001)$. Irrespective of the study group, a significant post-recovery decrease in CTL count was observed during both the 1st examination (Fig. 3d). During the 2nd examination, however, the post-recovery decrease was documented solely in the supplemented group. Nevertheless, the main effect of the supplementation on CTL count did not turn out to be statistically significant on ANOVA $(p=0.294)$.

The values of Treg to $(\mathrm{NK}+\mathrm{T} \delta \gamma+\mathrm{CTL})$ ratio are presented in Fig. 4a. No statistically significant changes in this parameter were documented during the 1st examination. During the 2nd examination, the post-recovery values of Treg to $(\mathrm{NK}+\mathrm{T} \delta \gamma+\mathrm{CTL})$ ratio in both supplemented group and placebo group turned out to be significantly higher than at the baseline. In line with these finding, ANOVA showed that the main effect of the supplementation on Treg to $(\mathrm{NK}+\mathrm{T} \delta \gamma+\mathrm{CTL})$ ratio was not statistically significant $(p=0.053)$.

Statistical analysis showed that the values of Treg to NK ratio were influenced by physical exercise $(p<0.001)$, but not by the supplementation $(p=0.233)$. Irrespective of the examination term, the post-exercise values of Treg to NK ratio in both study groups were lower than at the baseline, but this effect was not statistically significant (Fig. 4b). However, during the 2nd examination, the post-recovery Treg to NK ratio in the supplemented group turned out to be significantly higher than immediately after the exercise.

Exercise-induced changes in Treg to $T \delta \gamma$ ratio values are presented in Fig. 4c. This parameter turned out to be significantly modulated by strenuous physical exercise (main effect, $p<0.001$ ), but not by the LTE supplementation (main effect, $p=0.156$ ). The post-recovery increase in Treg to $\mathrm{T} \delta \gamma$ ratio values was observed during the 2nd examination, but only in the supplemented group.

Statistical analysis showed that the values of Treg to CTL ratio were modulated by the exercise, but not by the supplementation $(p<0.001$ and $p=0.771$, respectively; Fig. 4d). Irrespective of the study group, no statistically significant changes in Treg to CTL ratio were documented during the 1st examination. During the 2nd examination, a significant post-recovery increase in Treg to CTL ratio values was observed in both supplemented group and placebo group.

Selected indices of complete blood count and TAC levels determined during the 1st and the 2nd examination are shown in Table 5. Irrespective of the examination term, the exercise-induced changes in WBC counts in athletes from both groups followed a similar pattern, with a post-exercise increase in this parameter and its return to the baseline level after a 24-h recovery. During the 1st examination, physical exercise did not exert a significant effect on the percentages of lymphocytes and granulocytes. During the 2nd examination, a significant post-recovery increase in granulocyte percentage was observed in LTE-supplemented athletes, along with a significant post-recovery decrease in lymphocyte percentages in both groups.

Statistical analysis demonstrated that TAC was modulated solely by physical exercise $(p<0.001)$. Before the supplementation (1st examination), a significant post-exercise decrease in serum TAC was observed in both study groups. During the 2nd examination, a post-recovery increase in TAC level was observed in athletes from the supplemented group, but not in those from the placebo group (Table 5).

None of the athletes reported adverse events throughout the period of L-theanine supplementation.

\section{Discussion}

The present study, including members of Polish National Rowing Team, demonstrated that in the athletes supplemented with L-theanine, ergometer test contributed to a post-recovery decrease in IL-10 level (Fig. 1). The decrease in IL-10 level was associated with an increase in the values of IL-2 to IL-10 and IFN- $\gamma$ to IL-10 ratios. The post-recovery values of both ratios in the supplemented group were significantly higher than at the baseline (Fig. 2). These changes might correspond to a favorable shift in Th1/Th2 balance toward Th1 caused by supplementation with theanine, which would be consistent with our research hypothesis. Published evidence suggests that supplementation with theanine may influence concentration of some cytokines. Li et al. [24] demonstrated that both IL-4 to IFN- $\gamma$ ratio and serum concentrations of IL-10, IL- 6 and IL-4 decreased with a growing dose of L-theanine administered to rats via a feeding tube.

Since IL-10 inhibits the activity of Th1 cells and NK and decreases the synthesis of Th1 cytokines (IL-2 and 

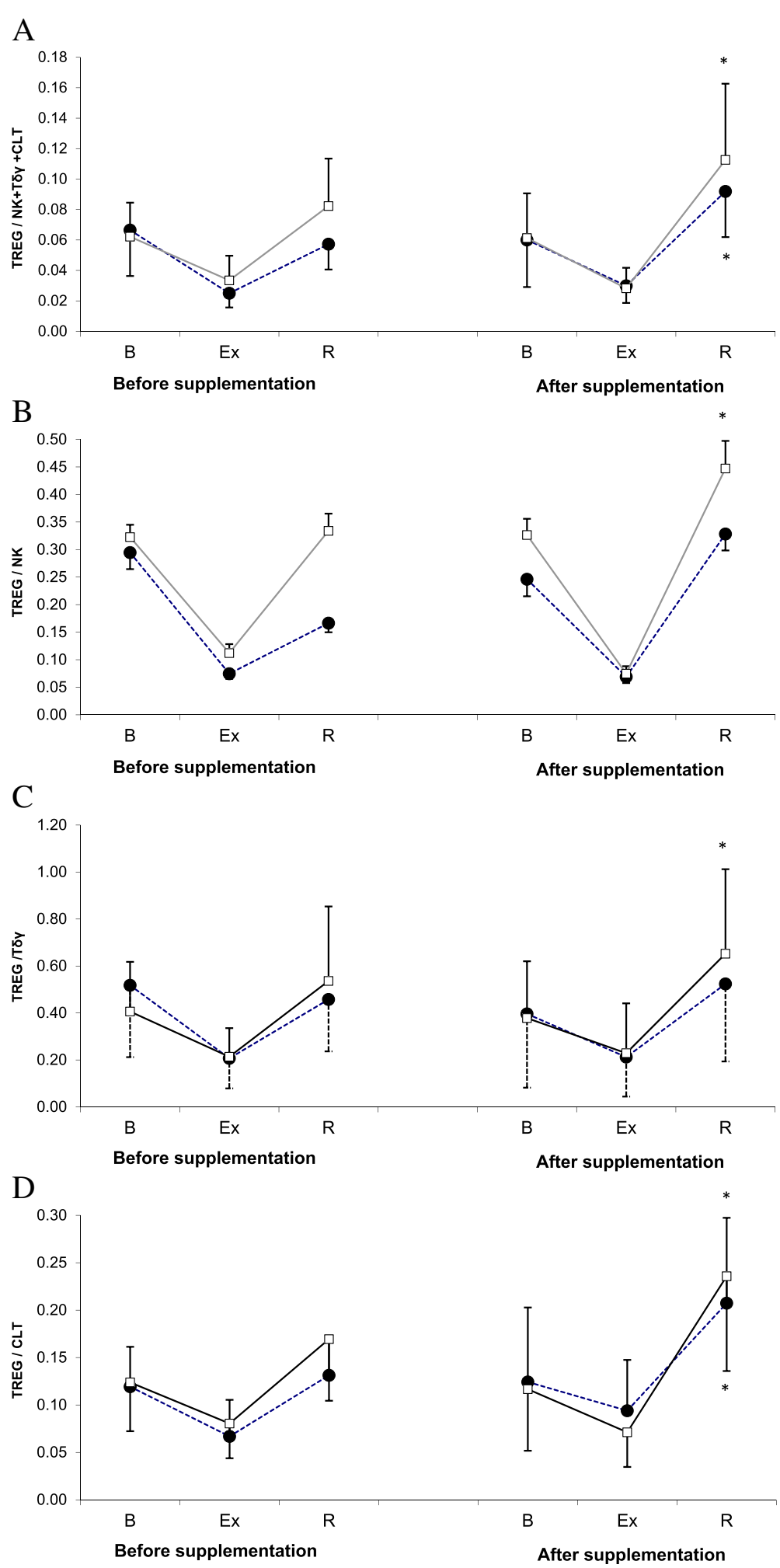

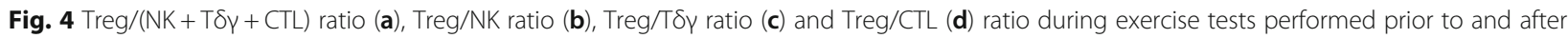
supplementation with LTE $(m e a n \pm S D)$. Note. Tregs = regulatory $T$ cells; NK = natural killer cells; $T \delta \gamma=$ gamma delta $T$ cells; $C T L=$ cytotoxic $T$ lymphocytes; $\square-$ SUPPL = supplemented group; $\bullet-$ PLA = placebo group; $B=$ baseline; $E x=$ post-exercise; $R=$ after a 1-day recovery; \# - significantly different compared to baseline level; * - significantly different compared to post-exercise level 
Table 5 Selected parameters of complete blood count and the level of total antioxidant capacity before and after supplementation with L-theanine (LTE) or placebo (Pla)

\begin{tabular}{|c|c|c|c|c|c|c|c|c|c|c|}
\hline \multicolumn{2}{|l|}{ Parameters } & \multicolumn{3}{|c|}{ Before supplementation } & \multicolumn{3}{|c|}{ After supplementation } & \multicolumn{3}{|l|}{ ANOVA } \\
\hline & & $\begin{array}{l}\text { Pre-Exercise } \\
x \pm S D\end{array}$ & $\begin{array}{l}\text { Post-Exercise } \\
x \pm S D\end{array}$ & $\begin{array}{l}\text { Post-Recovery } \\
x \pm S D\end{array}$ & $\begin{array}{l}\text { Pre-Exercise } \\
\mathrm{x} \pm \mathrm{SD}\end{array}$ & $\begin{array}{l}\text { Post-Exercise } \\
x \pm S D\end{array}$ & $\begin{array}{l}\text { Post-Recovery } \\
x \pm S D\end{array}$ & Exercise & LTE & $\overline{\text { Exercise } \times \text { LTE }}$ \\
\hline \multirow[t]{2}{*}{$\overline{W B C}\left[10^{9} / \mathrm{L}\right]$} & LTE & $6.81 \pm 1.47$ & $11.16 \pm 1.52^{*}$ & $6.53 \pm 1.04 t$ & $6.25 \pm 1.17$ & $11.12 \pm 1.96^{*}$ & $6.46 \pm 1.51^{\dagger}$ & $<0.0001$ & 0.2236 & 0.7378 \\
\hline & $\mathrm{Pla}$ & $5.98 \pm 1.27$ & $10.1 \pm 1.56^{*}$ & $5.75 \pm 0.75^{\dagger}$ & $6.03 \pm 1.13$ & $10.83 \pm 1.91^{*}$ & $6.0 \pm 0.74^{+}$ & & & \\
\hline \multirow[t]{2}{*}{ Granulocytes [\%] } & LTE & $46.0 \pm 10.81$ & $47.6 \pm 3.85$ & $52.6 \pm 5.53$ & $46.4 \pm 3.69$ & $41.9 \pm 5.37$ & $51.3 \pm 6.25^{\dagger}$ & $<0.0001$ & 0.8803 & 0.4637 \\
\hline & Pla & $48.9 \pm 5.68$ & $47.8 \pm 6.22$ & $48.8 \pm 5.44$ & $45.5 \pm 3.58$ & $43.4 \pm 7.35$ & $49.9 \pm 6.04$ & & & \\
\hline \multirow[t]{2}{*}{ Lymphocytes [\%] } & LTE & $42.73 \pm 7.85$ & $44.59 \pm 4.21$ & $38.44 \pm 6.04$ & $43.48 \pm 3.08$ & $48.36 \pm 4.93$ & $38.79 \pm 5.35^{\dagger}$ & $<0.0001$ & 0.5098 & 0.5920 \\
\hline & $\mathrm{Pla}$ & $43.19 \pm 5.32$ & $45.13 \pm 6.59$ & $42.83 \pm 6.02$ & $44.98 \pm 3.39$ & $47.62 \pm 6.99$ & $40.17 \pm 6.19^{\dagger}$ & & & \\
\hline \multirow[t]{2}{*}{ TAC (mmol/l) } & LTE & $2.36 \pm 0.38$ & $1.78 \pm 0.40^{*}$ & $2.11 \pm 0.55$ & $2.22 \pm 0.44$ & $1.80 \pm 0.35$ & $2.43 \pm 0.41^{\dagger}$ & $<0.0001$ & 0.2035 & 0.5617 \\
\hline & Pla & $2.49 \pm 0.22$ & $1.65 \pm 0.28^{*}$ & $2.18 \pm 0.42$ & $1.87 \pm 0.46$ & $1.69 \pm 0.24$ & $2.39 \pm 0.46$ & & & \\
\hline
\end{tabular}

WBC White blood cells; TAC Total Antioxidant Capacity

${ }^{*} p<0.05$ - compared to Pre-Exercise value

${ }^{\dagger} p<0.05$ - compared to Post-Exercise value

IFN- $\gamma$ ), its elevated concentrations may predispose to cellular immunosuppression [25-27]. In contrast, a decrease in IL-10 level boosts cellular immunity, which is vital not only in the context of URTI prevention but also for cancer control. One example of practical application of this knowledge is therapeutic suppression of IL-10 production with low-dose cyclophosphamide, which produces anti-metastatic effect due to a shift in Th1/Th2 balance toward Th1. In this context, previously published observations that IL-10 is a tumor growth factor and IFN- $\gamma$ exerts a cytotoxic effect on metastatic cells seem to be particularly worth emphasizing [28]. Thus, the increase in IFN- $\gamma$ to IL-10 ratio values observed after strenuous exercise in supplemented athletes but not in the controls seems to be particularly valuable finding.

However, the results of supplementation were different when the number of Tregs and some cytotoxic cells, NK, $\mathrm{T} \gamma \delta$ and CTL, were considered (Fig. 3). During the 2nd examination (after supplementation), the post-recovery Treg counts in both placebo group and supplemented group were significantly higher than at the baseline (Fig. 3a). Moreover, the post-recovery CTL count in athletes from the supplemented group was significantly lower than immediately after the exercise (Fig. 3d). Finally, during the 2nd examination, the post-recovery values of both Treg to NK and Treg to $T \gamma \delta$ ratios in the supplemented group were significantly higher than immediately after the exercise (Fig. 4b and c). The interpretation of these findings is not straightforward. Theoretically, the supplementation might contribute to a decrease in the number of cytotoxic CTLs. Also, the post-recovery increase in the values of Treg to NK and Treg to TCR ratios may suggest that the supplementation with LTE promoted unfavorable changes in the proportion of cytotoxic lymphocytes to Tregs. However, these results should not be interpreted without consideration of the cytokines synthesized by those cells, since otherwise, any conclusions about beneficial or unfavorable effect of LTE on immunity might be biased. A decrease in CTL count should be linked to a decrease in the level of IL-10 synthesized by Th2 cells, since according to literature, IL-10 promotes growth and differentiation of CTLs [29]. Equally important is the observation that the post-exercise increase in CTL count observed during the 2nd examination, although not statistically significant, was more evident in the supplemented group than in the controls. However, after a 24-h recovery, mean CTL counts in both groups were essentially the same.

Our study did not demonstrate an effect of LTE supplementation on $\mathrm{T} \gamma \delta$ count (Fig. 3c). It must be stressed, that the supplemented group and the placebo group did not differ significantly in terms of the concentrations of Th1 cytokines (IL-2 and IFN- $\gamma$ ) (Fig. 1a and d). However, the post-recovery number of CTLs (a source of Th1 cytokines) in the supplemented group was lower than immediately after the exercise (Fig. 3d). Thus, the decrease in IL-10 concentration and the increase in the values of IFN- $\gamma$ to IL-10 and IL-2 to IL-10 ratios after supplementation with LTE might reflect higher activity of $\mathrm{T} \gamma \delta$ cells in Th1 cytokine production. This is consistent with the results published by Mao et al. [30] who observed an inhibitory effect of IL-10 on the synthesis of Th1 cytokines by $\mathrm{T} \gamma \delta$ cells, as well as with the findings reported by Bukowski et al. [10] according to whom, LTE administered at lower concentrations enhanced production of Th1 cytokines (IFN- $\gamma, \mathrm{TNF}-\alpha$ ) by $\mathrm{T} \gamma \delta$ cells, and stimulated proliferation of those cells at higher concentrations.

In this study, we did not determine concentrations of other cytokines that theoretically could contribute to a shift in Th1/Th2 balance. Thus, we can only speculate that the post-supplementation decrease in CTL count and lack of changes in the number of other analyzed cytotoxic cells did not affect negatively cytotoxic potential of the 
athletes after the strenuous exercise. However, this hypothesis needs to be verified in further research.

Unlike Murakami et al. [11, 31], we did not observe statistically significant differences in the percentages of lymphocytes and granulocytes before and immediately after the ergometric test. Statistically significant changes, namely a decrease in the lymphocyte percentage in both groups and an increase in the percentage of granulocytes (the latter solely in the supplemented group) in relation to post-exercise values were observed no earlier than after the recovery (Table 5). In the study conducted by Murakami et al. [31], individuals from the control group showed a post-exercise increase in granulocyte percentage and a decrease in lymphocyte percentage, whereas a suppression of post-exercise neutrophilia and lymphopenia was observed in individuals supplemented with cystine and LTE. The discrepancies between our findings and the results published by Murakami et al. [31] might be associated with the use of different exercise loads. Nevertheless, the lack of post-exercise increase in granulocyte percentage and concomitant increase in lymphocyte percentage in the study conducted by those authors were most likely caused by cystine or its synergy with LTE. When administered separately, LTE caused an increase in granulocyte percentage, which was also observed in our present study (Table 5). According to Murakami et al. [31], the synergistic effect of cystine and LTE may contribute to an increase in glutathione concentration, which results in attenuation of oxidative stress, and thus, in lesser post-exercise neutrophilia and lymphopenia. It should be emphasized that also a training load specific for a given phase of the yearly cycle might exert an effect on the post-exercise changes in the study parameters (Table 2). In our present study, the strenuous ergometric test conducted before the supplementation (during the preparatory phase) did not induce any statistically significant changes in the percentages of granulocytes and lymphocytes. However, the change of training loads, namely an increase in the proportion of high-intensity exercises before the 2nd examination (competitive phase) was reflected by a post-recovery decrease in lymphocyte percentages, observed in both supplemented group and the controls (Table 5). It needs to be stressed that the competitive phase constitutes a serious strain for the athletes as despite a similar training volume $(970 \mathrm{~min}$ and 960 min during the 1st and 2nd examination, respectively), the proportion of high and very high intensity exercises is markedly larger (Table 2). High exercise intensity is an important element of adaptation to the so-called "competitive" loads, but may also exert an unfavorable effect contributing to an immune impairment [32].

Moreover, our study demonstrated that supplementation with theanine contributed to a statistically significant post-recovery increase in TAC, which implies that after the exercise, the redox potential in athletes from the supplemented group was higher than in the controls. In turn, the increase in the percentage of granulocytes observed in the supplemented group at the end of the recovery period might result from a decrease in the concentration of anti-inflammatory IL-10 and resultant increase in the level of granulocyte-macrophage stimulating factor (GM-CSF) (not analyzed in this study), stimulating proliferation and activity of granulocytes [33-35].

\section{Conclusions}

To the best of our knowledge, this was the first study to analyze the effect of L-theanine (LTE) on selected cytotoxic cells, Treg and some Th1 and Th2 cytokines in elite athletes exposed to strenuous exercise. After the 24-h recovery, athletes from the supplemented group presented with significantly lower CTL counts and significantly higher values of Treg to NK and Treg to CTL ratios than immediately after the exercise. Paradoxically, however, the post-recovery concentrations of IL-10 in LTE-supplemented athletes were significantly lower and the values of IFN- $\gamma$ to IL-10 and IL-2 to IL-10 ratios significantly higher than at the baseline. This implies that despite a decrease in CTL and unfavorable increase in Treg ratio to some cytotoxic cells, some elements of cellular immunity might be enhanced due to inhibition of IL-10 synthesis and a beneficial shift toward production of Th1 cytokines. Thus, it can be stipulated that despite the decrease in the number of some cytotoxic cells (CTLs) and an increase in the proportion of Tregs to CTLs observed after the supplementation, LTE may exert a beneficial effect on a disrupted Th1/Th2 balance, as shown by the decrease in IL-10 concentration. The decrease in IL-10 level, occurring regardless of higher TAC, might be a reason behind the post-recover increase in granulocyte percentage in the supplemented group.

\section{Abbreviations \\ CTL: Cytotoxic T lymphocyte; IL: Interleukin; INT-Y: Interferon gamma; LA: Lactic acid; NK: Natural killer; OTS: Overtraining syndrome; RDA: Recommended dietary allowance; TAC: Total antioxidant capacity;

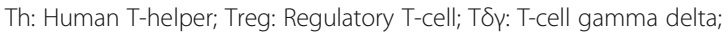 URTA: Upper respiratory tract infections; WBC: White blood cells}

\section{Acknowledgements}

We thank all subjects for cooperation with the research.

\section{Funding}

The experiment was supported from the grant No 0034/RS3/2015/53 funded by the Polish Ministry of Science and Higher Education.

\section{Availability of data and materials}

The datasets used and/or analyzed during the current study are available from the corresponding author on reasonable request.

\section{Authors' contributions}

The present study was designed by AJ and ASS; data were collected by $P B, J T$. KZ and KŁ; data interpretation and manuscript preparation were undertaken by AJ, ASS, EP, AG and BM. All authors approved the final version of the paper. 


\section{Ethics approval and consent to participate}

The experimental procedures and potential risks were explained to the participants, and a written informed consent was provided and signed prio to inclusion in the study. The study was conducted in accordance with the Declaration of Helsinki, and the protocol of the study was approved by the Local Bioethics Committee at the Poznan University of Medical Sciences (decision no. 769/13 of 10 October 2013).

\section{Consent for publication}

Not applicable.

\section{Competing interests}

The authors declare no conflict of interest, financial or otherwise. The authors declare that the results of the study are presented clearly, honestly and without fabrication, falsification or inappropriate data manipulation.

\section{Publisher's Note}

Springer Nature remains neutral with regard to jurisdictional claims in published maps and institutional affiliations.

\section{Author details}

'Department of Morphological and Health Sciences, Faculty of Physical Culture in Gorzów Wielkopolski, 13 Estkowskiego Str, 66-400 Gorzów Wielkopolski, Poland. ${ }^{2}$ Department of Water Sports, Faculty of Physical Culture in Gorzów Wielkopolski, 13 Estkowskiego Str, 66-400 Gorzów Wielkopolski, Poland. ${ }^{3}$ Department of Pediatrics, Endocrinology, Diabetology, Metabolic Disorders and Cardiology of Developmental Age, Pomeranian Medical University, Unii Lubelskiej Str, 71-252 Szczecin, Poland. ${ }^{4}$ Department of General Pathology, Pomeranian Medical University, 72 Powstanców Wielkopolskich Alley, 70-111 Szczecin, Poland. ${ }^{5}$ Faculty of Physical Culture in Gorzów Wielkopolski, 13 Estkowskiego Str, 66-400 Gorzów Wielkopolski, Poland.

\section{Received: 20 September 2018 Accepted: 6 February 2019}

\section{Published online: 15 February 2019}

\section{References}

1. Purvis D, Gonsalves S, Deuster PA. Physiological and psychological fatigue in extreme conditions: overtraining and elite athletes. PM\&R. 2010;2(5):442-50.

2. Smith DJ. A framework for understanding the training process leading to elite performance. Sports Med. 2003;33(15):1103-26.

3. Gleeson M, Pyne DB. Respiratory inflammation and infections in high-performance athletes. Immunol Cell Biol. 2016;94(2):124-31.

4. Handzlik M, Shaw A, Dungey M, Bishop N, Gleeson M. The influence of exercise training status on antigen-stimulated IL-10 production in whole blood culture and numbers of circulating regulatory T cells. Eur J Appl Physiol. 2013;113(7):1839-48.

5. Lakier Smith L. Overtraining, excessive exercise, and altered immunity: is this a T helper-1 versus T helper-2 lymphocyte response? Sports Med. 2003;33(5):347-64

6. Minuzzi L, Rama L, Bishop N, Rosado F, Martinho A, Paiva A, Teixeira AM. Lifelong training improves anti-inflammatory environment and maintains the number of regulatory T cells in masters athletes. Eur J Appl Physiol. 2017;117(6):1131-40

7. Tsuge H, Sano S, Hayakawa T, Kakuda T, Unno T. Theanine, gammaglutamylethylamide, is metabolized by renal phosphate-independent glutaminase. Biochim Et Biophys Acta. 2003;1620(1-3):47-53.

8. Kamath $A B$, Wang $L$, Das $H$, Li L, Reinhold VN, Bukowski JF. Antigens in tea-beverage prime human Vgamma 2Vdelta $2 \mathrm{~T}$ cells in vitro and in vivo for memory and nonmemory antibacterial cytokine responses. Proc Natl Acad Sci U S A. 2003;100(10):6009-14.

9. Wen H, Wei S, Zhang S, Hou D, Xiao W, He X. Effects of L-theanine on performance and immune function of yellow-feathered broilers. Chinese $J$ Anim Nutr. 2012;24:1946-54.

10. Bukowski JF, Morita CT, Brenner MB. Human gamma delta T cells recognize alkylamines derived from microbes, edible plants, and tea: implications for innate immunity. Immunity. 1999;11(1):57-65.

11. Murakami S, Kurihara S, Koikawa N, Nakamura A, Aoki K, Yosigi H, Sawaki K, Ohtani M. Effects of oral supplementation with cystine and theanine on the immune function of athletes in endurance exercise: randomized, double-blind, placebo-controlled trial. Biosci Biotechnol Biochem. 2009;73(4):817-21.

12. Kawada S, Kobayashi K, Ohtani M, Fukusaki C. Cystine and theanine supplementation restores high-intensity resistance exercise-induced attenuation of natural killer cell activity in well-trained men. J Strength Con Res. 2010;24(3):846-51.

13. Kurihara S, Shibahara S, Arisaka H, Akiyama Y. Enhancement of antigen-specific immunoglobulin G production in mice by co-administration of L-cystine and L-theanine. J Vet Med Sci. 2007;69(12):1263-70.

14. Romagnani S. The Th1/Th2 paradigm. Immunol Today. 2007;18(6):263-6.

15. Kaiko GE, Horvat JC, Beagley KW, Hansbro PM. Immunological decision-making how does the immune system decide to mount a helper T-cell response? Immunology. 2008;123(3):326-38.

16. Hansbro PM, Kaiko GE, Foster PS. Cytokine / anti-cytokine therapy - novel treatments for asthma? Br J Pharmacol. 2011;163(1):81-95.

17. Gao Q, Qiu S, Fan J, Zhou J, Wang X, Xiao Y, Xu Y, Li YW, Tang Z. Intratumoral balance of regulatory and cytotoxic $T$ cells is associated with prognosis of hepatocellular carcinoma after resection. J Clin Oncology. 2007;25(18):2586-93.

18. Kunzmann V, Kimmel B, Herrmann T, Einsele H, Wilhelm M. Inhibition of phosphoantigen-mediated gammadelta T-cell proliferation by CD4+ CD25+ FoxP3+ regulatory T cells. Immunology. 2009;126(2):256-67.

19. Sato E, Olson SH, Ahn J, Bundy B, Nishikawa H, Qian F, Jungbluth AA, Frosina D, Gnjatic S, Ambrosone C, Kepner J, Odunsi T, Ritter G, Lele S, Chen YT, Ohtani H, Old LJ, Odunsi K. Intraepithelial CD8+ tumorinfiltrating lymphocytes and a high CD8+/regulatory $T$ cell ratio are associated with favorable prognosis in ovarian cancer. Proc Natl Acad Sci U S A. 2005;102(51):18538-43.

20. Jarosz M. Nutrition standards for the polish population - amendment. Copyright by Instytut Żywności i Żywienia 2012 Warszawa.

21. Keenan EK, Rogers PJ, Priestley CM, Finnie MDA, Jones PS. How much theanine in a cup of tea? Effects of tea type and method of preparation. Food Chem. 2011;125(2):588-94.

22. Miyagawa K, Hayashi $Y$, Kurihara S, Maeda A. Co-administration of Lcystine and L-theanine enhances efficacy of influenza vaccination in elderly persons: nutritional status-dependent immunogenicity. Geriatr Gerontol Int. 2008:8:243-50.

23. Eschenauer G, Sweet BV. Pharmacology and therapeutic uses of theanine. Am J Health Syst Pharm. 2006;63(1):26-30.

24. Li C, Tong H, Yan Q, Tang S, Han X, Xiao W, Tan Z. L-Theanine improves immunity by altering $\mathrm{TH} 2 / \mathrm{TH} 1$ cytokine balance, brain neurotransmitters, and expression of phospholipase $C$ in rat hearts. Med Sci Monit. 2016;22:662-9.

25. Trinchieri G. Cytokines and cytokine receptors. Immunol Rev. 2004;202(1):5-7.

26. Bashyam H. Th1/Th2 cross-regulation and the discovery of IL-10. J Exp Med. 2007;204(2):237.

27. Chiu J, Ernst DM, Keating A. Acquired natural killer cell dysfunction in the tumor microenvironment of classic Hodgkin lymphoma. Front Immunol. 2018;9:267.

28. Matar P, Rozados VR, Gervasoni SI, Scharovsky GO. Th2/Th1 switch induced by a single low dose of cyclophosphamide in a rat metastatic lymphoma model. Cancer Immunol Immunother. 2002;50(11):588-96.

29. Chen WF, Zlotnik A. IL-10: a novel cytotoxic T cell differentiation factor. J Immunol. 1991;147(2):528-34.

30. Mao Y, Yin S, Zhang J, Hu Y, Huang B, Cui L, Kang N, He W. A new effect of IL-4 on human $\gamma \delta T$ cells: promoting regulatory $V \$ 1$ T cells via IL-10 production and inhibiting function of V $82 \mathrm{~T}$ cells. Cell Mol Immunol. 2016;13(2):217-28.

31. Murakami S, Kurihara S, Titchenal CA, Ohtani M. Suppression of exerciseinduced neutrophilia and lymphopenia in athletes by cystine/theanine intake: a randomized, double-blind, placebo-controlled trial. J Int Soc Sports Nutr. 2010;7(1):23.

32. Walsh NP, Gleeson M, Shephard RJ, Gleeson M, Woods JA, Bishop NC, Simon P. Position statement part one: immune function and exercise. Exerc Immunol Rev. 2011;17:6-63.

33. Geissler K, Ohler L, Födinger M, Virgolini I, Leimer M, Kabrna E, Kollars M, Skoupy S, Bohle B, Rogy M, Lechner K. Interleukin 10 inhibits growth and granulocyte/macrophage colony-stimulating factor production in chronic myelomonocytic leukemia cells. J Exp Med. 1996;184(4):1377-84. 
34. Tamassia N, Bianchetto-Aguilera F, Arruda-Silva F, Gardiman E, Gasperini S, Calzetti F, Cassatella MA. Cytokine production by human neutrophils: revisiting the "dark side of the moon". Eur J Clin Inves. 2018:e12952. https:// doi.org/10.1111/eci.12952.

35. Turner MD, Nedjai B, Hurst T, Pennington DJ. Cytokines and chemokines: at the crossroads of cell signalling and inflammatory disease. Biochim Biophys Acta. 2014;1843(11):2563-82.

Ready to submit your research? Choose BMC and benefit from:

- fast, convenient online submission

- thorough peer review by experienced researchers in your field

- rapid publication on acceptance

- support for research data, including large and complex data types

- gold Open Access which fosters wider collaboration and increased citations

- maximum visibility for your research: over $100 \mathrm{M}$ website views per year

At BMC, research is always in progress.

Learn more biomedcentral.com/submissions 